\title{
Bayesian Estimation of the Parameters of Discrete Weibull Type (I) Distribution
}

\author{
Samir Kamel Ashour \\ Cairo University, Cairo, Egypt, ashoursamir@hotmail.com \\ Mohamed Salem Abdelwahab Muiftah \\ Cairo University, Cairo, Egypt, mohamedmuftah1961@gmail.com
}

Follow this and additional works at: https://digitalcommons.wayne.edu/jmasm

Part of the Applied Statistics Commons, Social and Behavioral Sciences Commons, and the Statistical Theory Commons

\section{Recommended Citation}

Ashour, S. K., \& Muiftah, M. S. A. (2019). Bayesian estimation of the parameters of discrete Weibull type (I) distribution. Journal of Modern Applied Statistical Methods, 18(2), eP2740. doi: 10.22237/jmasm/ 1604189160

This Regular Article is brought to you for free and open access by the Open Access Journals at DigitalCommons@WayneState. It has been accepted for inclusion in Journal of Modern Applied Statistical Methods by an authorized editor of DigitalCommons@WayneState. 


\section{Bayesian Estimation of the Parameters of Discrete Weibull Type (I) Distribution}

\author{
Samir Kamel Ashour \\ Cairo University \\ Cairo, Egypt
}

\author{
Mohamed Salem Abdelwahab Muiftah \\ Cairo University \\ Cairo, Egypt
}

Bayesian estimation of the continuous Weibull distribution parameters was studied by Ahmad and Ahmad (2013) under the assumption of knowing the shape parameter. Bayesian estimates are considered here of the parameters of the discrete Weibull Type I [DW(I)] distribution and are obtained under two different assumptions: when the shape parameter is known, and when both parameters are independent random variables. A Mathcad program is performed to simulate data from the DW(I) distribution considering different values of the parameters and different sample sizes, and to obtain Bayesian parameter estimates. The resulted estimates are compared to the ML and proportion estimates obtained by Khan et al. (1989).

Keywords: Discrete Weibull distribution, Bayesian estimates, prior distribution, posterior distribution

\section{Introduction}

The discrete version of the Weibull distribution DW(I) was introduced by Nakagawa and Osaki (1975) as follows: If $T$ is a random variable following the continuous Weibull distribution with a shape parameter $\alpha>0$ and a scale parameter $\theta>0[T \sim \mathrm{W}(\alpha, \theta)]$, then the probability density function (pdf), the cumulative distribution function (cdf), the survival function (sf), and the failure rate (fr) of $T$ are, respectively:

doi: 10.22237/jmasm/1604189160 | Accepted: February 24, 2018; Published: July 24, 2020.

Correspondence: Mohamed Salem Abdelwahab Muiftah, mohamedmuftah1961@gmail.com 


$$
\begin{aligned}
& \mathrm{f}_{T}(t)=\alpha \theta t^{\alpha-1} \mathrm{e}^{-\theta t^{\alpha}}, \quad t>0 \\
& \mathrm{~F}_{T}(t)=\mathrm{P}(T \leq t)=1-\mathrm{e}^{-\theta t^{\alpha}} \\
& \mathrm{S}_{T}(t)=e^{-\theta t^{\alpha}} \\
& \mathrm{h}_{T}(t)=\alpha \theta t^{\alpha-1}
\end{aligned}
$$

Then, by inserting the sf given by (1) in the discretization formula

$$
\mathrm{P}(Y=y)=\mathrm{S}_{T}(y)-\mathrm{S}_{T}(y+1),
$$

the probability mass function (pmf) of the DW(I) distribution will be given by:

$$
\mathrm{P}(Y=y)=\mathrm{e}^{-\theta y^{\alpha}}-\mathrm{e}^{-\theta(y+1)^{\alpha}}, \quad y=0,1, \ldots
$$

By substituting $\mathrm{e}^{-\theta}=q$, the pmf, sf, and fr of the DW(I) are, respectively:

$$
\begin{aligned}
\mathrm{P}(Y=y) & =P_{y}=q^{y^{\alpha}}-q^{(y+1)^{\alpha}}, \quad 0<q<1, \quad y=0,1, \ldots \\
\mathrm{S}_{Y}(y) & =\mathrm{e}^{-\theta y^{\alpha}}=q^{y^{\alpha}} \\
\mathrm{h}_{Y}(q, \alpha) & =\frac{P_{y}}{\sum_{i=y}^{\infty} P_{i}}=1-q^{(y+1)^{\alpha}}-y^{\alpha}
\end{aligned}
$$

The first two moments of the DW(I) distribution are given by

$$
\mathrm{E}(Y)=\sum_{y=0}^{\infty} q^{y^{\alpha}} \quad \text { and } \quad \mathrm{E}(Y)=2 \sum_{y=0}^{\infty} y q^{y^{\alpha^{\alpha}}}+\mathrm{E}(Y) .
$$

Khan et al. (1989) compared the estimates of the parameters q and $\alpha$ obtained by method of moments and method of proportions. Kulasecara (1994) discussed the approximate ML estimation of the parameters under right censoring and compared his estimators with the proportion estimators introduced by Khan et al.

The pmf of the DW(I) distribution with parameters $q$ and $\alpha$ was also given by

$$
\mathrm{P}(Y=y)=q^{(y-1)^{\alpha}}-q^{y^{\alpha}}, \quad y=1,2, \ldots
$$


where $\alpha>0$ and $0<q<1$.

\section{Bayesian Estimation of the Parameters}

\section{Estimating Assuming that $\alpha$ is Known}

Given a random sample $Y_{1}, Y_{2}, \ldots, Y_{n}$ from the DW(I) distribution given by (2), then the likelihood function of this sample is

$$
\mathrm{L}(q \mid y)=\prod_{j=1}^{n}\left[q^{\left(y_{j}-1\right)^{\alpha}}-q^{y_{j}^{\alpha}}\right]
$$

According to the Jeffreys' rule (Jeffreys, 1946), the prior distribution $\mathrm{g}(u)$ of a given parameter $u$ is defined depending on its support as follows:

i. If $0<u<1$, then $\mathrm{g}(u) \propto \frac{1}{\sqrt{u(1-u)}}$;

ii. If $0<u<\infty$, then $\mathrm{g}(u) \propto \frac{1}{\sqrt{u}}$.

Thus, the prior distribution of $q$ may be considered to be

$$
\mathrm{g}(q) \propto \frac{1}{\sqrt{q(1-q)}}, \quad 0<q<1 .
$$

Then, the posterior distribution of $q$ is given by

$$
\begin{aligned}
\pi(q \mid y) \propto \mathrm{g}(q) \mathrm{L}(q \mid y) & \\
\propto & \frac{\prod_{j=1}^{n}\left[q^{\left(y_{j}-1\right)^{\alpha}}-q^{y_{j}^{\alpha}}\right]}{\sqrt{q(1-q)}}, \quad 0<q<1 \\
& =\frac{\prod_{j=1}^{n}\left[q^{\left(y_{j}-1\right)^{\alpha}}-q^{y_{j}^{\alpha}}\right]}{K \sqrt{q(1-q)}}, \quad 0<q<1
\end{aligned}
$$




\section{ASHOUR \& MUIFTAH}

The normalized constant (K) can be obtained by integrating (3) w.r.t. $q$ :

$$
K=\int_{0}^{1}\left\{\frac{\prod_{j=1}^{n}\left[q^{\left(y_{j}-1\right)^{\alpha}}-q^{y_{j}^{\alpha}}\right]}{\sqrt{q(1-q)}}\right\} d q .
$$

Thus, the posterior distribution of $q$ given $y$ will be

$$
\pi(q \mid y)=\frac{\prod_{j=1}^{n}\left[q^{\left(y_{j}-1\right)^{\alpha}}-q^{y_{j}^{\alpha}}\right]}{\sqrt{q(1-q)} \int_{0}^{1} \frac{1}{\sqrt{q(1-q)}} \prod_{j=1}^{n}\left[q^{\left(y_{j}-1\right)^{\alpha}}-q^{y_{j}^{\alpha}}\right] d q}, \quad 0<q<1
$$

Using squared error loss function, the Bayesian estimate and the Bayes' risk will be respectively, the mean and the variance of the posterior distribution (4). The mean and the variance of the posterior distribution of $q$ given $y$ are, respectively:

$$
\mathrm{E}(q \mid y)=\int_{0}^{1} q \times \pi(q \mid y) d q=\int_{0}^{1}\left\{\frac{q \prod_{j=1}^{n}\left[q^{\left(y_{j}-1\right)^{\alpha}}-q^{y_{j}^{\alpha}}\right]}{K \sqrt{q(1-q)}}\right\} d q
$$

and

$$
\begin{aligned}
\operatorname{Var}(q) & =\mathrm{E}\left(q^{2}\right)-[\mathrm{E}(q)]^{2} \\
& =\int_{0}^{1}\left\{\frac{q^{2} \prod_{j=1}^{n}\left[q^{\left(y_{j}-1\right)^{\alpha}}-q^{y_{j}^{\alpha}}\right]}{K \sqrt{q(1-q)}}\right\} d q-\left[\int_{0}^{1}\left\{\frac{q^{2} \prod_{j=1}^{n}\left[q^{\left(y_{j}-1\right)^{\alpha}}-q^{y_{j}^{\alpha}}\right]}{K \sqrt{q(1-q)}}\right\} d q\right]^{2}
\end{aligned}
$$

The mean given by (5) and the variance given by (6) need numerical techniques to be obtained. 


\section{BAYESIAN ESTIMATION OF DW(I) DISTRIBUTION'S PARAMETERS}

\section{Estimating Assuming that $q$ and $\alpha$ are Independent Random Variables}

According to the Jeffreys' rule and assuming that $q$ and $\alpha$ are independent, the prior distributions of $q$ and $\alpha$ may be considered to be

$$
\mathrm{g}_{1}(q) \propto \frac{1}{\sqrt{q(1-q)}} \quad \text { and } \quad \mathrm{g}_{2}(\alpha)=\frac{1}{\sqrt{\alpha}}
$$

Then, the joint prior distribution of $q$ and $\alpha$ is given by

$$
\pi(q, \alpha) \propto \mathrm{g}_{1}(q) \mathrm{g}_{2}(\alpha)=\frac{1}{\sqrt{\alpha q(1-q)}}, \quad 0<q<1, \quad \alpha>0 .
$$

The bivariate posterior distribution of $q$ and $\alpha$ is then given by

$$
\begin{aligned}
\pi(q, \alpha \mid y) \propto \pi(q, \alpha) \mathrm{L}(q, \alpha \mid y) & \\
\propto & \frac{\prod_{j=1}^{n}\left[q^{\left(y_{j}-1\right)^{\alpha}}-q^{y_{j}^{\alpha}}\right]}{\sqrt{\alpha q(1-q)}}, \quad 0<q<1, \quad \alpha>0 \\
& =\frac{\prod_{j=1}^{n}\left[q^{\left(y_{j}-1\right)^{\alpha}}-q^{y_{j}^{\alpha}}\right]}{C \sqrt{\alpha q(1-q)}}, \quad 0<q<1, \quad \alpha>0
\end{aligned}
$$

The normalized constant $(C)$ can be obtained by integrating (7) w.r.t. $q$ and $\alpha$ :

$$
C=\int_{0}^{\infty} \int_{0}^{1} \frac{\prod_{j=1}^{n}\left[q^{\left(y_{j}-1\right)^{\alpha}}-q^{y_{j}^{\alpha}}\right]}{\sqrt{\alpha q(1-q)}} d q d \alpha=\int_{0}^{1} \int_{0}^{\infty} \frac{\prod_{j=1}^{n}\left[q^{\left(y_{j}-1\right)^{\alpha}}-q^{y_{j}^{\alpha}}\right]}{\sqrt{\alpha q(1-q)}} d \alpha d q .
$$

The marginal posterior distribution of $q$ given $\alpha$ and $y$ is given by

$$
\pi_{1}(q \mid \alpha, y)=\int_{0}^{\infty}\left\{\frac{\prod_{j=1}^{n}\left[q^{\left(y_{j}-1\right)^{\alpha}}-q^{y_{j}^{\alpha}}\right]}{C \sqrt{\alpha q(1-q)}}\right\} d \alpha, \quad 0<q<1,
$$




\section{ASHOUR \& MUIFTAH}

and the marginal posterior distribution of $\alpha$ given $q$ and $y$ is given by

$$
\pi_{2}(\alpha \mid q, y)=\int_{0}^{1}\left\{\frac{\prod_{j=1}^{n}\left[q^{\left(y_{j}-1\right)^{\alpha}}-q^{y_{j}^{\alpha}}\right]}{C \sqrt{\alpha q(1-q)}}\right\} d q, \quad \alpha>0 .
$$

Using squared error loss function, the Bayesian estimate and the Bayes' risk will be the mean and the variance of the marginal posterior distribution respectively. The mean and the variance of the marginal posterior distributions of $q$ given $\alpha$ and $y$ are, respectively:

$$
\mathrm{E}(q \mid \alpha, y)=\int_{0}^{1} q\left\{\int_{0}^{\infty}\left(\frac{\prod_{j=1}^{n}\left[q^{\left(y_{j}-1\right)^{\alpha}}-q^{y_{j}^{\alpha}}\right]}{C \sqrt{\alpha q(1-q)}}\right) d \alpha\right\} d q
$$

and

$$
\begin{aligned}
\operatorname{Var}(q) & =\mathrm{E}\left(q^{2}\right)-[\mathrm{E}(q)]^{2} \\
& =\int_{0}^{1} q^{2}\left\{\int_{0}^{\infty}\left(\frac{\prod_{j=1}^{n}\left[q^{\left(y_{j}-1\right)^{\alpha}}-q^{y_{j}^{\alpha}}\right]}{C \sqrt{\alpha q(1-q)}}\right) d \alpha\right\} d q-[\mathrm{E}(q \mid \alpha, y)]^{2}
\end{aligned}
$$

The mean and the variance of the marginal posterior distributions of $\alpha$ given $q$ and $y$ are, respectively:

$$
\mathrm{E}(\alpha \mid q, y)=\int_{0}^{\infty} \alpha\left\{\int_{0}^{1}\left(\frac{\prod_{j=1}^{n}\left[q^{\left(y_{j}-1\right)^{\alpha}}-q^{y_{j}^{\alpha}}\right]}{C \sqrt{\alpha q(1-q)}}\right) d q\right\} d \alpha
$$

and 


\section{BAYESIAN ESTIMATION OF DW(I) DISTRIBUTION'S PARAMETERS}

$$
\begin{aligned}
\operatorname{Var}(\alpha) & =\mathrm{E}\left(\alpha^{2}\right)-[\mathrm{E}(\alpha)]^{2} \\
& =\int_{0}^{\infty} \alpha^{2}\left\{\int_{0}^{1}\left(\frac{\prod_{j=1}^{n}\left[q^{\left(y_{j}-1\right)^{\alpha}}-q^{y_{j}^{\alpha}}\right]}{C \sqrt{\alpha q(1-q)}}\right) d q\right\} d \alpha-[\mathrm{E}(\alpha \mid q, y)]^{2}
\end{aligned}
$$

The means given by (9) and (11) and the variances given by (10) and (12) need numerical techniques to be obtained.

\section{Simulation}

A Mathcad program is used to simulate data from DW(I) distribution for some values of $q$ and $\alpha$, three of them were used by Khan, et al. (1989), namely, $(q=0.6$, $\alpha=1.5),(q=0.8, \alpha=2.0)$, and $(q=0.5, \alpha=0.5)$, the Bayesian estimates of $q$ and $\alpha$ are obtained for sample sizes $(n=20,40,50,100)$ using 1000 replications, and compared to the ML and proportion estimates obtained by Khan et al.

\section{Results}

It is observed, in all studied cases, that the summation of the probabilities (the cdf) is not always the unity at a specific value of the random variable.

\section{Distribution I: $(q=0.6, \alpha=1.5)$}

Table 1 represents the pmf of the DW(I) $(1.5,0.6)$ (approximated to 10 decimals), which shows that the cdf reaches the value 1 when $Y=13$, that is, the probabilities of $Y=14, Y=15, \ldots$ and so on, are very small and may be ignored. The first two moments and the variance of the distribution are approximately given by

$$
\mathrm{E}(Y)=1.926873844, \mathrm{E}\left(Y^{2}\right)=4.667398731 \text {, and } \operatorname{Var}(Y)=0.9545559203 .
$$

\section{Distribution II: $(q=0.8, \alpha=0.2)$}

Table 2 represents the pmf of the $\mathrm{DW}(\mathrm{I})(0.2,0.8)$ (approximated to 10 decimals), which shows that the value 1 of the cdf is unreachable for any finite $Y$. The first two moments and the variance of the distribution are approximately given by

$$
\mathrm{E}(Y)=31941.19912, \mathrm{E}\left(Y^{2}\right)=13115582590 \text {, and } \operatorname{Var}(Y)=12095342390 .
$$


ASHOUR \& MUIFTAH

Table 1. The pmf of the DW(I) $(1.5,0.6)$

\begin{tabular}{rrr}
$\boldsymbol{y}$ & $\mathbf{P}(\boldsymbol{y})$ & $\mathbf{c d f}$ \\
\hline 1 & 0.4 & 0.4 \\
2 & 0.3642145624 & 0.7642145624 \\
3 & 0.1654393283 & 0.9296538907 \\
4 & 0.0535499493 & 0.9832038400 \\
5 & 0.0134874738 & 0.9966913138 \\
6 & 0.0027597743 & 0.9994510882 \\
7 & 0.0004710537 & 0.9999221419 \\
8 & 0.0000683052 & 0.9999904471 \\
9 & 0.0000085294 & 0.9999989765 \\
10 & 0.0000009270 & 0.9999999035 \\
11 & 0.0000000884 & 0.9999999919 \\
12 & 0.0000000075 & 0.9999999994 \\
13 & 0.0000000006 & 1.0000000000 \\
14 & $3.755306 \times 10^{-10}$ & 1.0000000000 \\
$\vdots$ & $\vdots$ & $\vdots$ \\
20 & $4.0898 \times 10^{-19}$ & 1.0000000000 \\
$\vdots$ & $\vdots$ & $\vdots$ \\
100 & $2.9542 \times 10^{-219}$ & 1.0000000000 \\
$\vdots$ & $\vdots$ & $\vdots$ \\
125 & $4.6784 \times 10^{-307}$ & 1.0000000000 \\
\hline
\end{tabular}

Table 2. The pmf of the $\operatorname{DW}(I)(0.2,0.8)$

\begin{tabular}{rrr}
$\boldsymbol{y}$ & $\mathbf{P}(\boldsymbol{y})$ & $\mathbf{c d f}$ \\
\hline 1 & 0.2 & 0.2 \\
2 & 0.0261093004 & 0.2261093004 \\
3 & 0.0165763282 & 0.2426856286 \\
$\vdots$ & $\vdots$ & $\vdots$ \\
100 & 0.0006429482 & 0.4290828972 \\
$\vdots$ & $\vdots$ & $\vdots$ \\
100000 & 0.0000004792 & 0.8926258176 \\
$\vdots$ & $\vdots$ & $\vdots$ \\
1000000 & 0.0000000206 & 0.9708874799 \\
$\vdots$ & $\vdots$ & $\vdots$ \\
$\infty$ & 0.0000000000 & 1.0000000000 \\
\hline
\end{tabular}

\section{Distribution III: $(q=0.5, \alpha=0.5)$}

Table 3 represents the pmf of the DW(I) $(0.5,0.5)$ (approximated to 10 decimals), and shows that the cdf reaches the value 1 when $Y=955$. The first two moments and the variance of the distribution are approximately given by

$$
\mathrm{E}(Y)=4.788218708, \mathrm{E}\left(Y^{2}\right)=108.6258571 \text {, and } \operatorname{Var}(Y)=85.6988187 .
$$




\section{BAYESIAN ESTIMATION OF DW(I) DISTRIBUTION'S PARAMETERS}

Table 3. The pmf of the DW(I) $(0.5,0.5)$

\begin{tabular}{rrr}
$\boldsymbol{y}$ & $\mathbf{P}(\boldsymbol{y})$ & $\mathbf{c d f}$ \\
\hline 1 & 0.5 & 0.5 \\
2 & 0.124785773 & 0.624785773 \\
$\vdots$ & $\vdots$ & $\vdots$ \\
10 & 0.013298355 & 0.888298355 \\
$\vdots$ & $\vdots$ & $\vdots$ \\
100 & $3.45264 \times 10^{-5}$ & 0.999023438 \\
$\vdots$ & $\vdots$ & $\vdots$ \\
955 & $\mathbf{5 . 6 1 8 1 5 \times 1 0 ^ { - 1 2 }}$ & $\mathbf{1 . 0 0 0 0 0 0 0 0 0}$ \\
956 & $5.55259 \times 10^{-12}$ & 1.000000000 \\
\hline
\end{tabular}

Table 4. The pmf of the DW(I) $(2.0,0.8)$

\begin{tabular}{rrr}
$\boldsymbol{y}$ & $\mathbf{P}(\boldsymbol{y})$ & $\mathbf{c d f}$ \\
\hline 1 & 0.2 & 0.2 \\
2 & 0.3904 & 0.5904 \\
3 & 0.2753822720 & 0.8657822720 \\
4 & 0.1060702303 & 0.9718525023 \\
5 & 0.0243696045 & 0.9962221068 \\
6 & 0.0034533746 & 0.9996754814 \\
$\vdots$ & $\vdots$ & $\vdots$ \\
10 & 0.0003066780 & 0.9999821594 \\
11 & 0.0000172129 & 0.9999993723 \\
12 & 0.0000006136 & 0.9999999859 \\
$\mathbf{1 3}$ & $\mathbf{0 . 0 0 0 0 0 0 0 1 3 9}$ & $\mathbf{1 . 0 0 0 0 0 0 0 0 0 0}$ \\
14 & 0.0000000002 & 1.0000000000 \\
$\vdots$ & $\vdots$ & $\vdots$ \\
57 & $1.2308 \times 10^{-8}$ & 1.00000000000 \\
58 & 0 & 1.00000000000 \\
\hline
\end{tabular}

\section{Distribution IV: $(q=0.8, \alpha=2.0)$}

Table 4 represents the pmf of the DW(I) $(2.0,0.8)$ (approximated to 10 decimals), and shows that the cdf reaches the value 1 when $Y=13$. The first two moments and the variance of the distribution are approximately given by

$$
\mathrm{E}(Y)=2.376, \mathrm{E}\left(Y^{2}\right)=6.687, \text { and } \operatorname{Var}(Y)=1.0416
$$

Table 5 represents the Bayesian estimates $\hat{q}_{\mathrm{B}}$ and $\hat{r}_{\mathrm{B}}$ of the scale parameter $q$ and the Bayes' risk $r$ of the suggested simulated DW(I) distributions for different sample sizes and their corresponding MSE's assuming that $\alpha$ is known. 


\section{ASHOUR \& MUIFTAH}

Table 5. Bayesian estimates of DW(I) parameters and their corresponding MSEs,

Case 1: $\alpha$ is known

\begin{tabular}{rrrrrrr}
$\boldsymbol{q}$ & $\boldsymbol{\alpha}$ & $\boldsymbol{n}$ & $\hat{\boldsymbol{q}}_{\mathrm{B}}$ & $\operatorname{MSE}\left(\hat{\boldsymbol{q}}_{\mathrm{B}}\right)$ & $\hat{\boldsymbol{r}}_{\mathrm{B}}$ & $\operatorname{MSE}\left(\hat{\boldsymbol{r}}_{\mathrm{B}}\right) \times \mathbf{1 0}^{-\mathbf{7}}$ \\
\hline 0.6 & 1.5 & 20 & 0.478 & 0.024 & 0.006 & 9.830 \\
& & 40 & 0.488 & 0.017 & 0.003 & 18.320 \\
& & 50 & 0.487 & 0.017 & 0.003 & 17.390 \\
& & 100 & 0.491 & 0.014 & 0.001 & 33.990 \\
0.5 & 0.5 & 20 & 0.461 & 0.009 & 0.006 & 1.986 \\
& & 40 & 0.474 & 0.005 & 0.003 & 0.707 \\
& & 50 & 0.471 & 0.004 & 0.003 & 0.140 \\
& & 100 & 0.475 & 0.002 & 0.001 & 1.427 \\
0.8 & 0.2 & 20 & 0.606 & 0.039 & 0.004 & 1.251 \\
& & 40 & 0.609 & 0.037 & 0.002 & 0.310 \\
& & 50 & 0.608 & 0.037 & 0.002 & 0.203 \\
& & 100 & 0.609 & 0.037 & 0.001 & 0.525 \\
0.8 & 2.0 & 20 & 0.703 & 0.015 & 0.003 & 8.799 \\
& & 40 & 0.717 & 0.009 & 0.002 & 3.003 \\
& 50 & 0.715 & 0.009 & 0.002 & 12.540 \\
& & 100 & 0.722 & 0.007 & 0.001 & 238.300 \\
\hline
\end{tabular}

Table 6. The Bayesian estimates of DW(I) parameters and their corresponding MSEs, Case 2: both $\alpha$ and $q$ are unknown

\begin{tabular}{rrrrrrrrr}
$\boldsymbol{q}$ & $\boldsymbol{\alpha}$ & $\boldsymbol{n}$ & $\hat{\boldsymbol{q}}_{\mathrm{B}}$ & $\operatorname{MSE}\left(\hat{\boldsymbol{q}}_{\mathrm{B}}\right)$ & $\hat{\boldsymbol{\alpha}}_{\mathrm{B}}$ & $\operatorname{MSE}\left(\hat{\boldsymbol{\alpha}}_{\mathrm{B}}\right)$ & $\hat{\boldsymbol{r}}_{\mathrm{B}}$ & $\operatorname{MSE}\left(\hat{\boldsymbol{r}}_{\mathrm{B}}\right) \times \mathbf{1 0}^{-6}$ \\
\hline 0.6 & 1.5 & 20 & 0.431 & 0.039 & 3.534 & 84.959 & 0.010 & 2.060 \\
& & 40 & 0.430 & 0.035 & 1.726 & 2.833 & 0.006 & 13.480 \\
& & 50 & 0.425 & 0.035 & 1.577 & 0.299 & 0.005 & 28.530 \\
& & 100 & 0.424 & 0.034 & 1.468 & 0.143 & 0.001 & 913.400 \\
0.5 & 0.5 & 20 & 0.446 & 0.013 & 0.268 & 0.074 & 0.010 & 46.592 \\
& & 40 & 0.449 & 0.008 & 0.268 & 0.081 & 0.005 & 9.573 \\
& & 50 & 0.445 & 0.007 & 0.221 & 0.084 & 0.004 & 6.324 \\
& & 100 & 0.441 & 0.005 & 0.200 & 0.091 & 0.002 & 1.383 \\
0.8 & 0.2 & 20 & 0.883 & 0.013 & 0.442 & 0.182 & 0.003 & 21.977 \\
& & 40 & 0.901 & 0.017 & 0.405 & 0.112 & 0.002 & 11.333 \\
& & 50 & 0.896 & 0.015 & 0.373 & 0.073 & 0.003 & 6.943 \\
& 100 & 0.909 & 0.084 & 0.347 & 0.064 & 0.001 & 2.248 \\
0.8 & 2.0 & 20 & 0.612 & 0.046 & 2.973 & 8.326 & 0.010 & 19.620 \\
& & 40 & 0.616 & 0.040 & 2.525 & 0.893 & 0.006 & 32.740 \\
& 50 & 0.613 & 0.040 & 2.464 & 0.727 & 0.004 & 53.580 \\
& 100 & 0.614 & 0.037 & 2.337 & 0.341 & 0.001 & 887.200 \\
\hline
\end{tabular}




\section{BAYESIAN ESTIMATION OF DW(I) DISTRIBUTION'S PARAMETERS}

Table 6 represents the Bayesian estimates $\hat{q}_{\mathrm{B}}, \hat{\alpha}_{\mathrm{B}}$, and $\hat{r}_{\mathrm{B}}$ of the scale parameter $q$, shape parameter $\alpha$, and the Bayes' risk $r$ of the suggested simulated DW(I) distributions for different sample sizes and their corresponding MSE's assuming that $q$ and $\alpha$ are independent random variables.

From Tables 5 and 6 it may observed that the Bayesian estimates of the parameters $q, \alpha$, and $r$ become closer to the true parameter values as the sample size increases in most cases, which may implies the consistency of these estimates, the MSE's of the estimates become also smaller as the sample size increases in most cases. Tables 7 and 8 represent the estimates of the parameters $q$ and $\alpha$ of the DW(I) distributions, respectively, and their corresponding variances obtained by Khan et al. (1989) accompanied with our Bayesian estimates.

An inspection of Tables 7 and 8 shows that the estimates obtained by the method of moments seem to be more close to the true parameter values in most cases, it seems also that the variances of the Bayesian estimates of the scale parameter $q$ are smaller in most cases than those of other estimates.

Table 7. The estimates of the parameter $q$ of $\mathrm{DW}(\mathrm{I})$ and their corresponding variances

\begin{tabular}{|c|c|c|c|c|c|c|c|c|c|c|}
\hline \multirow[b]{3}{*}{$q$} & \multirow[b]{3}{*}{$\alpha$} & \multirow[b]{3}{*}{$n$} & \multirow{2}{*}{\multicolumn{2}{|c|}{$\begin{array}{l}\text { Method of } \\
\text { moments }\end{array}$}} & \multirow{2}{*}{\multicolumn{2}{|c|}{$\begin{array}{l}\text { Method of } \\
\text { proportions }\end{array}$}} & \multicolumn{4}{|c|}{ Bayesian estimates } \\
\hline & & & & & & & \multicolumn{2}{|c|}{$\alpha$ is known } & \multicolumn{2}{|c|}{$a$ is unkown } \\
\hline & & & $\hat{q}_{M}$ & $\operatorname{Var}(\hat{q} \mathrm{M})$ & $\hat{q}_{P}$ & $\operatorname{Var}\left(\hat{q}_{\mathrm{P}}\right)$ & $\hat{q}_{\mathrm{B}}$ & $\operatorname{Var}\left(\hat{q}_{\mathrm{B}}\right)$ & $\hat{q}_{B}$ & $\operatorname{Var}\left(\hat{q}_{B}\right)$ \\
\hline \multirow{4}{*}{0.6} & 1.5 & 20 & 0.615 & 0.013 & 0.603 & 0.014 & 0.478 & 0.009 & 0.431 & 0.011 \\
\hline & & 40 & 0.604 & 0.006 & 0.598 & 0.006 & 0.488 & 0.005 & 0.430 & 0.006 \\
\hline & & 50 & 0.601 & 0.005 & 0.596 & 0.005 & 0.487 & 0.004 & 0.425 & 0.005 \\
\hline & & 100 & 0.604 & 0.002 & 0.603 & 0.002 & 0.491 & 0.002 & 0.424 & 0.003 \\
\hline \multirow[t]{4}{*}{0.5} & 0.5 & 20 & 0.616 & 0.045 & 0.443 & 0.014 & 0.461 & 0.0008 & 0.453 & 0.010 \\
\hline & & 40 & 0.590 & 0.032 & 0.437 & 0.007 & 0.474 & 0.0034 & 0.449 & 0.005 \\
\hline & & 50 & 0.571 & 0.024 & 0.434 & 0.005 & 0.471 & 0.0029 & 0.445 & 0.006 \\
\hline & & 100 & 0.551 & 0.013 & 0.432 & 0.003 & 0.475 & 0.0014 & 0.441 & 0.001 \\
\hline \multirow[t]{4}{*}{0.8} & 2.0 & 20 & 0.797 & 0.007 & 0.788 & 0.010 & 0.703 & 0.0058 & 0.612 & 0.010 \\
\hline & & 40 & 0.796 & 0.004 & 0.791 & 0.005 & 0.717 & 0.0041 & 0.616 & 0.006 \\
\hline & & 50 & 0.766 & 0.003 & 0.792 & 0.004 & 0.715 & 0.0020 & 0.613 & 0.005 \\
\hline & & 100 & 0.801 & 0.001 & 0.800 & 0.002 & 0.722 & 0.0012 & 0.614 & 0.003 \\
\hline
\end{tabular}


Table 8. The estimates of the parameter $\alpha$ of $\mathrm{DW}(\mathrm{I})$ and their corresponding variances

\begin{tabular}{|c|c|c|c|c|c|c|c|c|}
\hline \multirow[b]{2}{*}{$q$} & \multirow[b]{2}{*}{$\alpha$} & \multirow[b]{2}{*}{$n$} & \multicolumn{2}{|c|}{$\begin{array}{l}\text { Method of } \\
\text { moments }\end{array}$} & \multicolumn{2}{|c|}{$\begin{array}{l}\text { Method of } \\
\text { proportions }\end{array}$} & \multicolumn{2}{|c|}{$\begin{array}{l}\text { Bayesian } \\
\text { estimates }\end{array}$} \\
\hline & & & $\hat{a}_{\mathrm{M}}$ & $\operatorname{Var}\left(\hat{\alpha}_{M}\right)$ & $\hat{\alpha}_{\mathrm{p}}$ & $\operatorname{Var}\left(\hat{\alpha}_{\mathrm{p}}\right)$ & $\hat{\boldsymbol{\alpha}}_{\mathrm{B}}$ & $\operatorname{Var}\left(\hat{\alpha}_{\mathrm{B}}\right)$ \\
\hline \multirow[t]{4}{*}{0.6} & 1.5 & 20 & 2.116 & 0.219 & 1.565 & 0.248 & 3.534 & 80.823 \\
\hline & & 40 & 2.046 & 0.107 & 1.510 & 0.127 & 1.726 & 2.738 \\
\hline & & 50 & 2.035 & 0.085 & 1.503 & 0.100 & 1.577 & 0.293 \\
\hline & & 100 & 2.040 & 0.042 & 1.528 & 0.050 & 1.468 & 0.142 \\
\hline \multirow[t]{4}{*}{0.5} & 0.5 & 20 & 0.693 & 0.052 & 0.542 & 0.131 & 0.268 & 2.429 \\
\hline & & 40 & 0.623 & 0.034 & 0.493 & 0.062 & 0.268 & 0.008 \\
\hline & & 50 & 0.580 & 0.032 & 0.477 & 0.042 & 0.221 & 0.008 \\
\hline & & 100 & 0.531 & 0.011 & 0.488 & 0.018 & 0.200 & 0.001 \\
\hline \multirow[t]{4}{*}{0.8} & 2.0 & 20 & 2.116 & 0.219 & 2.074 & 0.637 & 2.973 & 7.380 \\
\hline & & 40 & 2.046 & 0.107 & 2.030 & 0.330 & 2.525 & 0.617 \\
\hline & & 50 & 2.035 & 0.085 & 2.005 & 0.234 & 2.464 & 0.511 \\
\hline & & 100 & 2.040 & 0.042 & 2.039 & 0.109 & 2.337 & 0.227 \\
\hline
\end{tabular}

\section{References}

Ahmad, S. P., \& Ahmad, K. (2013). Bayesian analysis of Weibull

distribution using R software. Australian Journal of Basic and Applied Sciences, 7(9), 156-164. Retrieved from http://www.ajbasweb.com/old/ajbas/2013/July/156-164.pdf

Jeffreys, H. (1946). An invariant form for the prior probability in estimation problems. Proceedings of the Royal Society A, 186(1007), 453-461. doi: 10.1098/rspa.1946.0056

Khan, M. S. A., Khalique, A., \& Aboummoh, A. M. (1989). On estimating parameters in a discrete Weibull distribution. IEEE Transactions on Reliability, 38(3), 348-350. doi: 10.1109/24.44179

Kulasecara, K. B. (1994). Approximate MLEs of the parameters of a discrete Weibull distribution with type-I censored data. Microelectronics Reliability, 34(7), 1185-1188. doi: 10.1016/0026-2714(94)90502-9

Nakagawa, T., \& Osaki, S. (1975). The discrete Weibull distribution. IEEE Transactions on Reliability, R-24(5), 300-301. doi: 10.1109/TR.1975.5214915 